\title{
Death by cat bite: Pasteurella multocida: Autopsy findings and review of the literature
}

\author{
William Humphrey, Jessica W. Crothers, Anne M. Stowman, Sharon L. Mount \\ Pathology and Laboratory Medicine, University of Vermont Medical Center, Burlington, VT, USA
}

Received: May 11, 2020

Accepted: June 9, 2020

Online Published: June 20, 2020

DOI: $10.5430 /$ crcp.v7n1p11

URL: https://doi.org/10.5430/crcp.v7n1p11

\begin{abstract}
Pasteurella multocida, a gram-negative bacillus, is present in the oropharyngeal secretions of livestock, wild animals, and domesticated pets and can cause infection in humans. The most common route of entry has been shown to be via an animal bite, but a significant portion of cases of human infection lack evidence of such a wound. Review of the literature reveals that patients with a history of an animal bite tend to have a less aggressive clinical course than patients without an animal source of infection. We present a case, however, of Pasteurella multocida bacteremia which resulted in the death of an immunocompromised 80-year old woman in which the route of infection was found at autopsy to be a cat bite. This case highlights the importance of educating patients, particularly those with underlying immunocompromised conditions, of the possible lethal complications that can result from animal inflicted wounds and the importance of seeking medical assistance should a bite occur.
\end{abstract}

Key Words: Animal bite, Infectious, Pasteurella multocida, Autopsy

\section{INTRODUCTION}

Pasteurella multocida is a small, pleomorphic, nonflagellated, gram-negative coccobacillus commonly found in the upper respiratory and gastrointestinal tracts of livestock, wild, and domesticated animals. In humans, P. multocida infection most commonly results from bites, licks on broken human skin, or direct contact with oral secretions from a pet. While not every bite is clinically significant, Pasteurella species are isolated from $75 \%$ of cat bite wounds and $50 \%$ of dog bite wounds. ${ }^{[1]}$ The severity of P. multocida infections can range from localized infection to bacteremia (40\%-63\% of all patients), pneumonia, meningitis (17\%-29\% of all patients), and death. ${ }^{[2]}$ Studies have shown that Pasteurellosis has an overall mortality rate of $30 \% .{ }^{[3]}$ While immunocompromised patients have a higher rate of systemic infection and mortality, they are less likely to present with evidence of a bite wound. ${ }^{[1,4,5]}$ We present a case of Pasteurella multocida bacteremia causing the death of an 80-year old immunocompromised female. While oropharyngeal colonization was the clinically suspected route of entry, a bite wound was ultimately discovered at autopsy.

\section{Case Presentation}

\subsection{History}

The patient was an 80-year old woman who presented to the emergency department with a complaint of a sore throat. Her past medical history included hypertension, depression and anxiety, dementia, cerebrovascular disease, and thrombocytopenia and leukopenia, which were not further defined despite prior hematologic consultation. Evaluation, including radiologic imaging of the neck, did not demonstrate airway edema or abscess. The patient was instructed on basic care of

\footnotetext{
*Correspondence: William Humphrey; Email: william.humphrey@uvmhealth.org; Address: 111 Colchester Ave, Courtyard at Given S269, Burlington, VT 05401, USA.
} 
typical acute pharyngitis and encouraged to follow-up with her primary care provider in 2-3 days.

Two days after onset of symptoms, the patient returned to the emergency department with dyspnea and the report of an unwitnessed syncopal event. Although afebrile she required oxygen and became hypotensive. The patient was treated with an intravenous bolus of crystalloid and intravenous norepinephrine infusion. She was started on azithromycin, ceftriaxone, and vancomycin and admitted to the medical intensive care unit for management of septic shock and hypoxemic respiratory failure. Viral testing was negative. Blood cultures were obtained.

Laryngoscopy showed a patent airway without asymmetric swelling and purulent drainage of the larynx and hypopharynx. Admission blood cultures returned positive after 7 hours demonstrating growth of gram-negative bacilli and metronidazole therapy was initiated. Speciation of the organism by Matrix-assisted laser desorption/ionization time-of-flight (MALDI-TOF) revealed it to be Pasteurella multocida. At that point, azithromycin, vancomycin, and metronidazole were discontinued and the patient remained on intravenous ceftriaxone. Further clinical investigation revealed that the patient owned a cat. As an external wound-site for potential source of infection (feline bite) was not identified, it was hypothesized by the clinical team that the source of infection was oropharyngeal colonization.

On hospital day three the patient was persistently hypoglycemic, requiring intravenous dextrose infusion and her hypoxemia continued to worsen. After failing to improve, the patient was transitioned to comfort care and died on hospital day 8, 10 days following initial presentation.

\subsection{Autopsy findings}

External examination revealed evidence of a $1.5 \mathrm{~cm} \times 0.5 \mathrm{~cm}$ laceration and several small abrasions to the left lower leg adjacent to the medial malleolus (see Figure 1). Microscopic exam of tissue at this site showed surface ulceration with reepithelialization. Collections of neutrophils were seen within the dermis, and areas of degenerative changes. Vessel wall thickening and calcifications were also present. No thrombi were identified (see Figure 2).

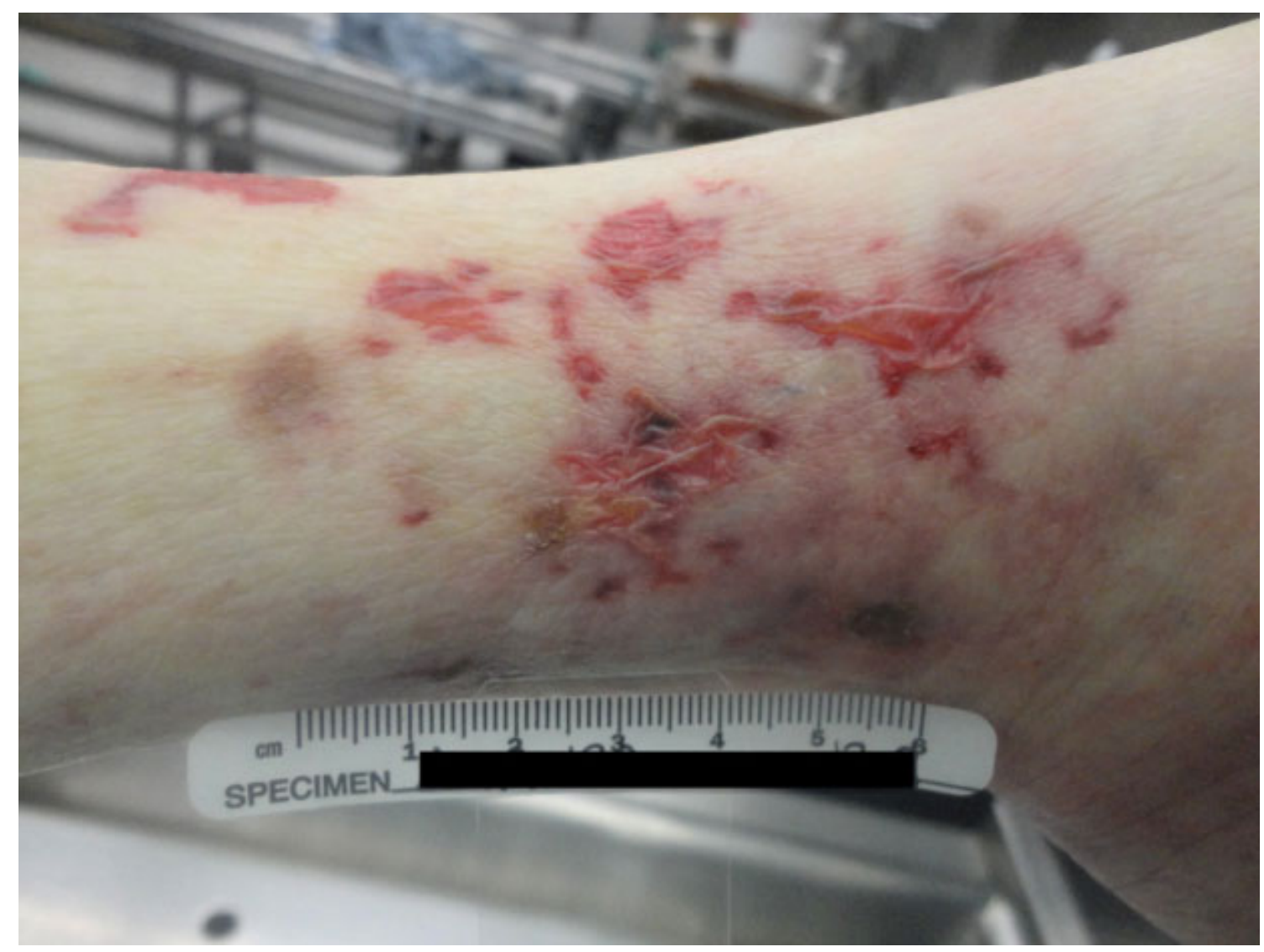

Figure 1. Left lower extremity wound, suspected animal bite 


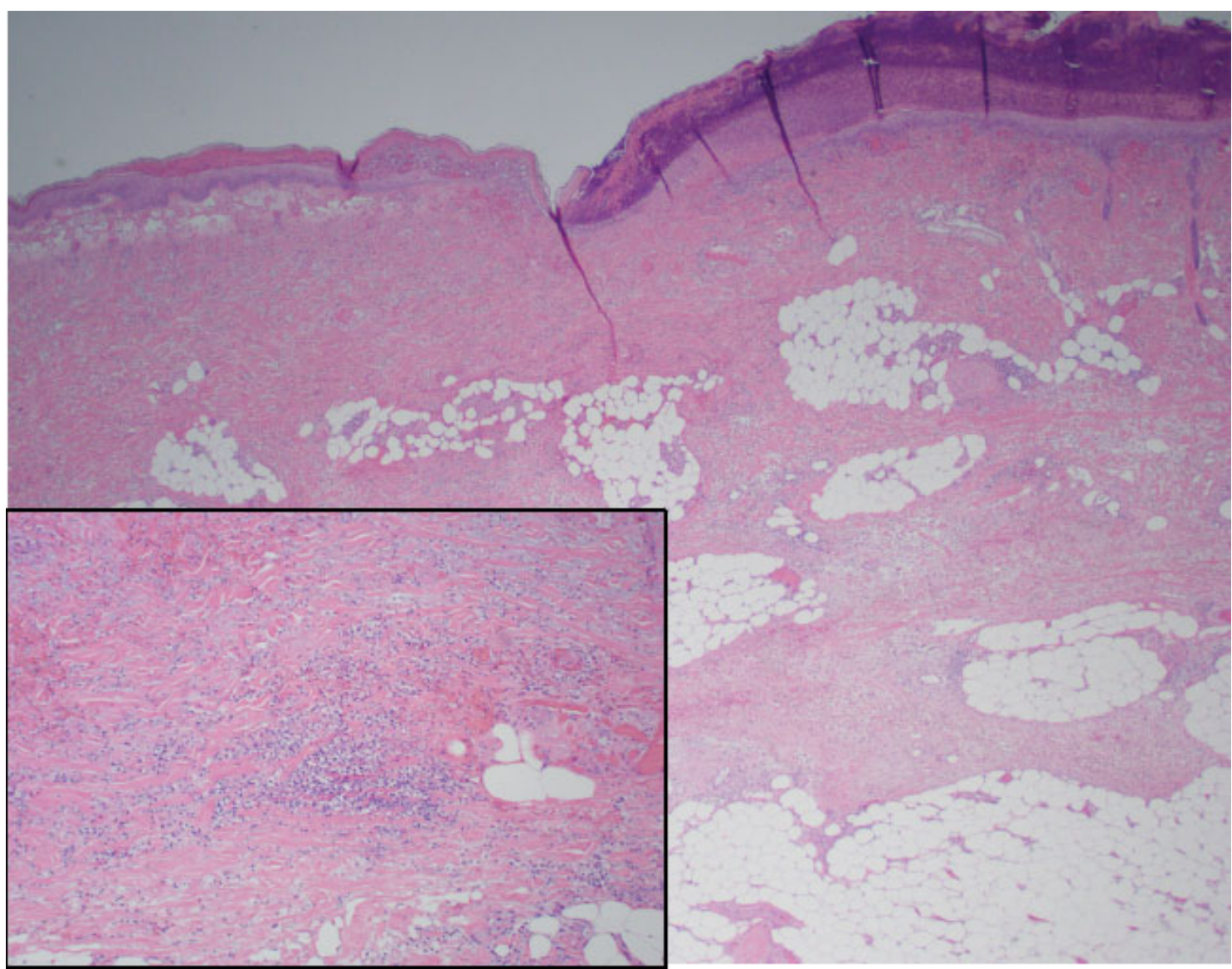

Figure 2. Low-power examination of the skin biopsy from the lower leg shows focal epidermal ulceration with neutrophilic scale crust, epidermal necrosis, and partial reepithelialization. Within the dermis, there is mixed perivascular and interstitial inflammation, extending into the subcutaneous tissue. (H\&E, 2x). Insert: On high-power, collections of neutrophils and neutrophilic debris, along with red cell extravasation, are seen within the mid dermis. The vessels show congestion and degenerative changes. A modified gram stain (not shown) did not show bacterial organisms within the skin biopsy. (H\&E, 10x)

Gross examination of the lungs revealed features of acute pneumonia with areas of consolidation. Examination of the laryngopharynx revealed an edematous airway, particularly in the area of the epiglottis. Multiple hematoxylin and eosin stained histologic sections from all lobes of the lung revealed airspace neutrophils consistent with acute pneumonia. Histologic modified gram stain and Periodic Acid Schiff (PAS), were negative for micro-organisms.

Other autopsy findings included pathologic changes associated with hypertensive and atherosclerotic cardiovascular disease and extensive necrosis of the liver. Neuropathologic examination documented remote lacunar infarcts as well as moderate arteriolosclerosis.

\section{Discussion}

Our patient presented initially with complaints of odynophagia and shortness of breath prior to identification of Pasteurella multocida by blood cultures. The clinical team sus- pected that her airway may have been colonized by the bacteria, however evidence of an animal bite was identified at the left lower extremity during post-mortem examination. Additionally, autopsy examination revealed findings of acute pneumonia, presumably secondary to Pasteurella bacteremia, coinciding with her clinical course of increasing hypoxia and oxygen requirement. Additionally, hepatic findings of extensive necrosis are consistent with end organ failure due to sepsis. Regardless of route of pathogen entry, appropriate antibiotic treatment had been initiated immediately upon pathogen identification.

Carriage rates of $\mathrm{P}$. multocida are highest in cats and dogs, up to $90 \%$ and $50 \%$ respectively. ${ }^{[5,6]}$ While asymptomatic colonization of humans by P. multocida has also been documented in the literature, severe disease is reported in colonized patients with underlying pulmonary disease. ${ }^{[1]}$ Alternatively, the majority of P. multocida infections in humans are in the context of direct animal inoculation (e.g. bite) ex- 
posure. ${ }^{[1,3]}$ While the histologic differential of the leg lesion includes ischemic injury, the lack of other foci of ischemic dermal changes throughout the patient's body, including on her other leg, favor that although missed at the time of admission, this wound likely represents a cat bite. Lack of bacteria identified on modified gram stain is consistent with the patient's history of prior antibiotic therapy. In addition, the patient's clinical course is consistent with Pasteurella multocida infection resulting in bacteremia and sepsis.

The literature suggests that patients with Pasteurella infection without evidence of a cat bite have a worse prognosis. These patients are more likely to have severe underlying comorbidities, including an immunocompromised state, and have higher rates of ICU admission and ultimately, mortality. ${ }^{[1,5,7-9]}$ It may be instructive that our patient was diagnosed with leukopenia and thrombocytopenia only a few weeks before her death. One can speculate that an underlying immunodeficiency increased her risk of systemic infection while also decreasing her body's ability to mount a successful local immune response, allowing the normal signs and symptoms of a local infection to be missed. Of note, at least one author has suggested that patients with specific conditions and immunocompromise of any kind should be dissuaded from owning pets due to the increased risk of severe morbidity and mortality related to P. multocida infection. ${ }^{[10]}$

Our case is unusual in its rapidly fatal course without identification of a portal of entry. Although P. multocida was identified as the infectious pathogen in the patient, this case highlights the importance of identifying a bite wound early in the course of a Pasteurella infection, particularly in patient populations at increased risk for severe complications. For patients with underlying immunocompromise, education and regular surveillance for pet-inflicted wounds may be indicated.

\section{ACKNOWLEDGements}

Our special thanks to Donald Dukette and Rachel Martindale of the University of Vermont Medical Center Department of Pathology and Laboratory Medicine.

\section{CONFLicts OF InTEREST Disclosure}

We declare that we have no conflict interests.

\section{REFERENCES}

[1] Giordano A, Dincman T, Clyburn BE, et al. Clinical Features and Outcomes of Pasteurella multocida Infection. Medicine (Baltimore) 2015; 94(36): e1285. PMid:26356688. https ://doi.org/10.109 7/MD. 0000000000001285

[2] Wilson BA, Ho M. Pasteurella multocida: from zoonosis to cellular microbiology. Clin Microbiol Rev. 2013; 26(3): 631-655. PMid:23824375. https : //doi.org/10.1128/CMR.00024-13

[3] Kofteridis DP, Christofaki M, Mantadakis E, et al. Bacteremic community-acquired pneumonia due to Pasteurella multocida. Int J Infect Dis. 2009 May; 13(3): e81-3. Epub 2008 Oct 8 PMid:18845465. https://doi.org/10.1016/j.ijid.2008.06 .023

[4] Zarlasht F, Khan M. A Case of Recurrent Pasteurella Bacteremia in an Immunocompetent Patient with No Animal Bite. Am J Case Rep. 2018; 19: 95-98. Published in 2018 Jan 25. PMid:29367585. https://doi.org/10.12659/AJCR.907251

[5] Abreu F, Rodríguez-Lucas C, Rodicio MR, et al. Human Pasteurella multocida Infection with Likely Zoonotic Transmission from a Pet Dog, Spain. Emerg Infect Dis. 2018; 24(6): 1145-46 PMid:29774848. https ://doi.org/10.3201/eid2406.171998

[6] Owen CR, Buker EO, Bell JF, et al. Pasteurella multocida in animals' mouths. Rocky Mt Med J. 1986; 65: 45-46.

[7] Narsana N, Farhat F. Septic shock due to Pasteurella multocida bacteremia: a case report. J Med Case Rep. 2015; 9: 159. Published 2015 Jul 11. PMid:26163266. https ://doi .org/10.1186/s132 56-015-0643-3

[8] Caserza L, Piatti G, Bonaventura A, et al. Sepsis by Pasteurella multocida in an Elderly Immunocompetent Patient after a Cat Bite. Case Rep Infect Dis. 2017;2017:2527980. PMid:29318064. https://doi.org/10.1155/2017/2527980

[9] Yamamoto T, Umehara T, Murase T, et al. Pasteurella multocida Septicemia in a Patient with Cirrhosis: An Autopsy Report. Case Rep Infect Dis. 2015;2015:597806. PMid:25692055. https : //doi. org/10.1155/2015/597806

[10] Olshtain-Pops K, Yinnon AM. Pasteurella multocida sepsis-should immunocompromised patients give up their pets? Israel Med Assoc J. 2008; 10: 648-649. 